\title{
Could Inbred Cases Identified in GWAS Data Succeed in Detecting Rare Recessive Variants Where Affected Sib-Pairs Have Failed?
}

\author{
Emmanuelle Génin ${ }^{a-c}$ Mourad Sahbatou ${ }^{d}$ Steven Gazal ${ }^{a, b}$ \\ Marie-Claude Babron ${ }^{a, b}$ Hervé Perdry ${ }^{e} f$ Anne-Louise Leutenegger ${ }^{a, b}$ \\ anserm UMR-946, Genetic Variability and Human Diseases, and ' Institut Universitaire d'Hématologie, \\ Université Paris Diderot, Paris, 'Inserm UMR-1078, Génétique, Génomique Fonctionnelle et Biotechnologies, Brest,

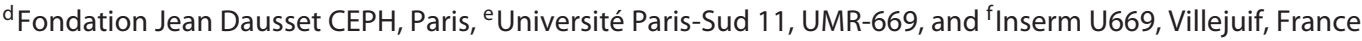

\section{Key Words}

Rare recessive variants · Inbreeding · Association · Linkage · Genome-wide association studies

\begin{abstract}
To detect fully penetrant rare recessive variants that could constitute Mendelian subentities of complex diseases, we propose a novel strategy, the HBD-GWAS strategy, which can be applied to genome-wide association study (GWAS) data. This strategy first involves the identification of inbred individuals among cases using the genome-wide SNP data and then focuses on these inbred affected individuals and searches for genomic regions of shared homozygosity by descent that could harbor rare recessive disease-causing variants. In this second step, analogous to homozygosity mapping, a heterogeneity lod-score, HFLOD, is computed to quantify the evidence of linkage provided by the data. In this paper, we evaluate this strategy theoretically under different scenarios and compare its performances with those of linkage analysis using affected sib-pair (ASP) data. If cases affected by these Mendelian subentities are not enriched in the sample of cases, the HBD-GWAS strategy has almost no power to detect them, unless they explain an important part of the disease prevalence. The HBD-GWAS strategy outperforms the ASP linkage strategy only in a very limited number
\end{abstract}

of situations where there exists a strong allelic heterogeneity. When several rare recessive variants within the same gene are involved, the ASP design indeed often fails to detect the gene, whereas, by focusing on inbred individuals using the HBD-GWAS strategy, the gene might be detected provided very large samples of cases are available.

Copyright $\odot 2013$ S. Karger AG, Base

\section{Introduction}

Genome-wide association studies (GWAS) have helped evidence the role of several common genetic variants in complex diseases, but taken together these common variants only explain a small part of the heritability of these traits [1]. Different candidates have been suggested to explain this missing heritability and among them are rare variants [2]. The SNP chips used to perform GWAS contain SNPs that were selected to tag most of the common variants present in human populations at a frequency of at least $5 \%$ but are not designed to directly capture rare variants. However, some information on these rare variants might be gained by using analysis methods other than the single marker tests that are usually used to analyze GWAS data.

\section{KARGER}

E-Mail karger@karger.com

www.karger.com/hhe
C 2013 S. Karger AG, Basel

0001-5652/12/0744-0142\$38.00/0 
A first approach consists in testing for association with the haplotypes formed by the alleles at nearby SNPs within a genomic region. Such an approach was developed by Zhu et al. [3] and applied on the Wellcome Trust Case Control Consortium (WTCCC) data to evidence 8 novel regions associated with some of the traits that are likely to harbor rare variants [4]. In a recent paper, Browning and Thompson [5] suggested another approach that consists in searching for regions of the genome where cases share alleles identical-by-descent (IBD) more often than controls. IBD mapping strategies were developed in the nineties to search for genetic risk factors in founder populations $[6,7]$, since with the sparse maps of microsatellite markers that were available at that time only long IBD segments could be detected. With dense SNP maps, it is now possible to detect IBD segments as small as $2 \mathrm{cM}$ and IBD mapping can thus be used in outbred populations [8]. Browning and Thompson [5] performed extensive simulations to determine under which conditions IBD mapping is efficient at detecting the effect of rare variants. They found that the strategy is more powerful than single marker association tests when multiple rare causal variants are clustered within a gene, but the required sample sizes still remain prohibitive under most of the scenarios investigated.

The aforementioned approaches have focused on rare variants with dominant effects and not considered the case of rare recessive variants. Recessive variants, however, are also likely to play an important role in diseases. Indeed, it has long been known, from mutagenesis studies in many different diploid organisms, that the majority (over 90\%) of mutations are recessive to the wild type (for a review, see [9]). Interestingly, through a functional classification of the proteins encoded by 923 disease genes, Jimenez-Sanchez et al. [10] found that the diseases caused by genes coding for enzymes were predominantly recessive and were also the largest functional category represented in their dataset, accounting for $31.2 \%$ of the total. Disease genes affected by recessive mutations are also less conserved than those affected by dominant mutations and this could probably be due to the fact that recessive mutations could remain hidden from selection while at the heterozygous state [11]. Similarly, Blekhman et al. [12] found more widespread and stronger purifying selection on genes associated with dominant rather than recessive phenotypes. Taken together, these different characteristics of recessive mutations make them good candidates for being disease-causing mutations.

The power of association tests to detect the effect of recessive alleles is often limited as these tests usually as-

Rare Recessive Variant Detection in GWAS Data sume an additive model that performs especially poorly for low-frequency recessive causal alleles [13]. This could probably partially explain why, in the literature, there are more reports of risk alleles with dominant effects than with recessive effects. Turning to rare recessive alleles involved in monogenic traits, the strategy of choice to detect them is homozygosity mapping [14]. Homozygosity mapping consists in focusing on inbred affected individuals and searching for a region of the genome of shared homozygosity. The method has been very successful in identifying rare recessive variants involved in several Mendelian disorders [15] but requires that the genealogy of patients be known so that inbred patients can be identified and their respective inbreeding coefficient, F, estimated. Leutenegger et al. $[16,17]$ proposed estimating inbreeding coefficients from genome-wide genetic data and performing homozygosity mapping on isolated inbred cases. A lod-score, referred to as FLOD, similar to Morton's lod-score [18], is computed based on the estimated $\mathrm{F}$ and the observed genotypes at markers. This method has recently been successful in identifying a new locus involved in Taybi-Linder syndrome [19].

In this paper, we investigate the possibility to use homozygosity mapping on case-only data from GWAS to evidence rare recessive variants potentially involved in the disease. The idea to use homozygosity mapping-like strategies on GWAS case-control data is not new and was first advocated by Gibbs and Singleton [20] as one of the possible applications of genome-wide SNP typing beyond simple association tests. Different methods were then developed to identify runs of homozygosity ( $\mathrm{ROHs}$ ) in the genome of cases and controls and to compare their distribution and localization (for a review, see [21]). A first successful application of $\mathrm{ROH}$ analysis was the detection of highly penetrant recessive loci in schizophrenia [22]. The problem, however, with $\mathrm{ROH}$ analysis is to determine if the observed homozygosity is due to inbreeding, in which case it is often referred to as homozygosity by descent (HBD), or if it is only due to the presence of frequent haplotypes in the studied population. Wang et al. [23] and more recently Zhang et al. [24] have introduced methodological developments to identify, among the different ROHs detected, those that are more likely due to inbreeding and could carry rare recessive mutations involved in disease susceptibility. However, it is not clear under which disease scenarios these approaches will be efficient at detecting rare recessive variants and how they compare to other linkage approaches and in particular to approaches based on IBD sharing in affected sib-pairs (ASP). We are particularly interested 
here in situations where rare variants are fully penetrant, but there exists some extreme heterogeneity with many different variants within the gene possibly involved in the disease. Could homozygosity mapping identify such monogenic-like disease subentities that ASP studies could have missed? To help answer this question, we propose, in this paper, a new strategy to identify genomic regions likely to harbor rare recessive variants, evaluate its performances under different disease models with fully penetrant rare recessive variants and compare them to the performances of ASP linkage analysis. An application is also presented on the publicly available type- 2 diabetes data from the WTCCC.

\section{Material and Methods}

\section{The HBD-GWAS Strategy}

To evidence rare recessive variants in a given gene $G$ involved in a disease, we propose to focus on the inbred affected individuals from a case-control sample genotyped for GWAS and to search for a region of the genome that a portion of them share HBD using a strategy similar to homozygosity mapping: the HBD-GWAS strategy.

In a first step, the inbreeding coefficient of each individual is estimated using FEstim [17] on the SNP data. To avoid bias due to the presence of linkage disequilibrium between the SNP alleles, estimates are computed on several sparse submaps as in Leutenegger et al. [25]. We then select all $I$ individuals with a median estimated inbreeding coefficient above a given threshold $T$ and search for linkage. The choice of this threshold $T$ is made in order to ensure that we select only individuals who are truly inbred and who will be informative for linkage. In most applications, we found that choosing $T=0.01$ appears a good compromise, since it ensures that only inbred individuals are selected without reducing their number too much. For each selected individual $i$, each marker $m$ on each submap $s$, a lod-score $\operatorname{FLOD}^{(i)}(m, s)$ is computed using the same equation as in Leutenegger et al. [16]:

$$
\operatorname{FLOD}^{(i)}(m, s)=\log _{10} \frac{P\left(X_{m, s}^{(i)}=1 \mid Y^{(i)}\right)+q^{\prime} \cdot P\left(X_{m, s}^{(i)}=0 \mid Y_{m, s}^{(i)}\right)}{\hat{f}_{s}^{(i)}+q^{\prime} \cdot\left(1-\hat{f}_{s}^{(i)}\right)}
$$

with the following parameters:

- $q^{\prime}$ : the assumed frequency of the mutant allele involved in the disease for this individual;

- $X_{m, s}^{(i)}$ : the HBD status of individual $i$, at marker $m$ on submap $s$ that is estimated together with $m, s$ : the inbreeding coefficient using the hidden Markov model of FEstim;

- $Y_{m, s}^{(i)}$ : the observed genotype of individual $i$, at marker $m$ on submap $s$

- $\hat{f}_{s}^{(i)}$ : the estimated inbreeding coefficient of individual $i$ on submap s.

Results are then averaged over the different submaps to obtain a single $F L O D^{(i)}(m)$ at each marker $m$.

Linkage evidence is evaluated over the entire set of $I$ inbred affected individuals by computing a lod-score, $\operatorname{HFLOD}(m, \alpha)$, at each marker $m$, with a heterogeneity parameter $\alpha$ that takes into account the possibility that only a fraction of the inbred affected individuals carry disease-causing alleles in gene $G$ :

$$
\begin{aligned}
& \operatorname{HFLOD}(m, \alpha)= \\
& \sum_{i=1}^{I} \log _{10}\left[\alpha \cdot \exp \left(\operatorname{FLOD}^{(i)}(m) \times \ln (10)\right)+(1-\alpha)\right] .
\end{aligned}
$$

This heterogeneity lod-score is then maximized over $\alpha$ to evaluate the evidence of linkage at marker $m$ and estimate the proportion of cases linked to this locus.

\section{Expected Power of the HBD-GWAS Strategy}

Let us consider a disease $D$ with prevalence $K$ that can be due to the effect of $x$ fully penetrant rare recessive variants $M_{\mathrm{i}}(i=1$, $\ldots, x)$ within gene $G$. Given the very low frequency of the different variants, it is very unlikely that two of them have arisen on the same haplotype, and thus we assume that two different variants cannot be carried on the same haplotype (in cis). Under this assumption, individuals cannot carry more than two disease-causing variants within the gene $G$. This is in fact equivalent to assuming that there is a single disease-causing locus within gene $G$ with $x+1$ alleles. For simplicity, it is also assumed that each of the $x$ variants $M_{i}$ has the same frequency $q^{\prime}=q / x$ and the remaining allele, A, has frequency $(1-q)$ in the sample population. Each individual can be in any of these following genotype categories (GC):

- GC 1: homozygous $\mathrm{M}_{\mathrm{i}} \mathrm{M}_{\mathrm{i}}$ for any of the $x$ disease-causing variants $\mathrm{M}_{\mathrm{i}}$ with probability $P_{1}=(q / x)^{2}$;

- GC 2: heterozygous $\mathrm{M}_{\mathrm{i}} \mathrm{M}_{\mathrm{j}}$ for two different disease-causing variants $\mathrm{M}_{\mathrm{i}}$ and $\mathrm{M}_{\mathrm{j}}$ (with $\mathrm{j} \neq \mathrm{i}$ ) with probability $P_{2}=2(q / x)^{2}$;

- GC 3: heterozygous $\mathrm{M}_{\mathrm{i}} \mathrm{A}$ with one disease-causing variant $\mathrm{M}_{\mathrm{i}}$ and the non-disease-causing allele $\mathrm{A}$ with probability $P_{3}=$ $2(q / x)(1-q)$;

- GC 4: homozygous AA with probability $P_{4}=(1-q)^{2}$.

The above probabilities $P_{i}(i=1-4)$ are derived under the assumption that the sample population is panmictic. Now, let us assume that, in the sample population, a proportion $\gamma$ of the individuals are inbred and, for simplicity, all have the same inbreeding coefficient $F$. These 4 probabilities now write:

$$
\begin{aligned}
& P_{1}(\gamma)=(q / x)^{2}+\gamma F(q / x)(1-(q / x)) ; \\
& P_{2}(\gamma)=2(1-\gamma F)(q / x)^{2} ; \\
& P_{3}(\gamma)=2(1-\gamma F)(q / x)(1-q) ; \\
& P_{4}(\gamma)=\gamma F(1-q)+(1-\gamma F)(1-q)^{2} .
\end{aligned}
$$

We consider two different disease models. In the first disease model, referred to as composite recessive model (COMPO_REC), individuals are affected with disease $D$ with a probability of 1 , if they carry two variants (complete penetrance), and with a probability of $f_{0}$ (phenocopy rate), if they carry less than two variants. In the second disease model, referred to as true recessive model (TRUE_REC), only individuals homozygous for a same variant have a probability of 1 of being affected and individuals heterozygous with two different disease-causing variants have a probability of $f_{0}$ of being affected. Given the prevalence constraint and the fact that there are, respectively, $E_{1}=x, E_{2}=x(x-1) / 2, E_{3}=x$ and $E_{4}=$ 1 possible genotypes in each of the 4 genotype categories defined above, $f_{0}$ can be written as a function of $K, q, F, \gamma$ and $x$ using the following equations: 
Under the TRUE_REC model:

$$
K=x P_{1}(\gamma)+f_{0}\left[\frac{x(x-1)}{2} P_{2}(\gamma)+x P_{3}(\gamma)+P_{4}(\gamma)\right]
$$

and thus,

$$
f_{0}=\frac{K-q\left[\frac{q}{x}-\gamma F\left(1+\frac{q}{x}\right)\right]}{(1-\gamma F)\left(1-\frac{q^{2}}{x}\right)+\gamma F(1-q)}
$$

Under the COMPO_REC model:

$$
K=x P_{1}(\gamma)+\frac{x(x-1)}{2} P_{2}(\gamma)+f_{0}\left[x P_{3}(\gamma)+P_{4}(\gamma)\right]
$$

and thus,

$$
f_{0}=\frac{K+q[(1-\gamma F) q-\gamma F]}{(1-\gamma F)\left(1-q^{2}\right)+\gamma F(1-q)}
$$

Note that, since $f_{0}$ cannot be negative, $q$ should not exceed some limits, $\mathrm{q}_{\text {lim,TRUE_REC }}$ and $\mathrm{q}_{\text {lim,COMPO_REC, }}$ respectively, which are determined by solving the following equations:

for qlim,TRUE_REC:

$x P_{1}(\gamma)-K=0$

and for qlim,COMPO_REC:

$x P_{1}(\gamma)+\frac{x(x-1)}{2} P_{2}(\gamma)-K=0$.

The genotype relative risk (GRR) of the variants is easily derived from $f_{0}$ since $\mathrm{GRR}=1 / f_{0}$.

If we now focus on the inbred individuals, their expected number in a sample of $N$ random individuals is simply $\gamma \times N$. In a sample of $N$ individuals affected by the disease, it is $I=N \times T$, where $T$ is the probability of being inbred given affected:

$$
\begin{aligned}
T & =P(\text { inbred } \mid \text { affected }) \\
& =\frac{\gamma}{K} \sum_{i=1}^{4} E_{i} \times P(\text { affected } \mid G C=i) \times P(G C=i \mid \text { inbred })
\end{aligned}
$$

with $E_{i}$ being the number of possible genotypes in GC $i$ (i.e. $x$, $x(x-1) / 2, x$ and 1 for $i=1-4$, respectively) and the probabilities $P(G C=i \mid$ inbred $)$ being obtained by setting $\gamma=1$ in the $P_{i}(\gamma)$ equations given above.

Among these affected inbred individuals, we are interested in determining how many of them are expected to have inherited two IBD variants in gene $G$ and are thus likely to share an HBD region around the gene. Only affected individuals in the first GC (GC= 1) can have inherited one variant IBD with probability

$$
R=\frac{\gamma F}{\gamma F+(1-\gamma F) \frac{q}{x}} .
$$

The expected number $H$ of inbred cases with an HBD region around gene $G$, referred to as HBD cases for short, is then

$$
\begin{aligned}
H & =N \times P(H B D \mid \text { affected }) \\
& =N \times \frac{\gamma F q}{K} .
\end{aligned}
$$

Interestingly, when we assume that all variants are equifrequent, $H$ depends only on the cumulative variant frequency and not on the number $(x)$ of variants within gene $G$. It is the same under the two disease models considered.

If we assume that among the $I$ inbred affected individuals only the $H$ individuals with two homozygous-by-descent variants contribute to the lod-score, the expected maximum EHFLOD values that can be reached at a marker completely linked to gene $G$ is then:

$$
\text { EHFLOD }=H \times \log _{10}\left(\frac{H}{I} \times \frac{1}{F}+\left(1-\frac{H}{I}\right)\right) .
$$

Indeed, since we have assumed complete penetrance, the likelihood of an individual $i$ having an HBD region around gene $G$ under hypothesis $H_{1}$ that 'the studied locus is linked to the disease' is 1. The likelihood under the null hypothesis $H_{0}$ of 'no linkage' is the probability for the individual to be $\mathrm{HBD}$ in a random region of the genome which is in fact the inbreeding coefficient $\mathrm{F}$. This will be equivalent to considering in equation 1 that $P\left(X_{m, s}^{(i)}=1 \mid Y^{(i)}\right)=1$ and thus $P\left(X_{m, s}^{(i)}=0 \mid Y^{(i)}\right)=0$ and that the frequency $q^{\prime}$ of the variant is small enough for $q^{\prime}\left(1-\hat{f}_{s}^{(i)}\right)$ to be negligible compared to $\hat{f}_{s}^{(i)}$.

This EHFLOD lod-score is a multipoint heterogeneity lodscore and its distribution under $H_{0}$ is

$$
\frac{1}{2} \chi_{0}^{2}+\frac{1}{2} \chi_{1}^{2}
$$

where $\chi_{0}^{2}$ is a distribution degenerated at zero with the probability of 1 and $\chi_{1}^{2}$ is the 1 degree-of-freedom $\chi^{2}$ distribution [26]. The expected power associated with an EHFLOD value can thus be obtained by using non-central $\chi^{2}$ distributions.

\section{Expected Power of the ASP Strategy}

Under the two disease models considered, we also computed the expected power to detect linkage if ASP were used. Only outbred sib-pairs are considered here. We assume that the IBD status of the alleles of the two sibs within each sib-pair is known without ambiguity. The expected distribution $\left(Z_{0}, Z_{1}, Z_{2}\right)$ of sib-pairs with 0,1 or 2 alleles IBD, respectively, is computed by listing all possible ASP and deriving their respective probabilities as a function of the different parameters $x, q$ and $f_{0}$ using our own script. The expected power to detect linkage on a sample of $N_{A S P}$ ASP is then derived by comparing this expected distribution against the distribution expected under $H_{0}$ using non-central $\chi^{2}$ as in Génin and ClergetDarpoux [27]. A nominal type- 1 error rate of $2.2 \times 10^{-5}$ is considered here, as this was the level required to declare significant linkage in Lander and Kruglyak's guidelines [28].

To compare with the EHFLOD, the expected maximum lodscore (EMLS) proposed by Risch in 1990 [29] is also computed, using the same equation as in Poznik et al. [30]:

$$
\begin{aligned}
& E M L S= \\
& N_{A S P} \times\left[Z_{0} \log _{10}\left(4 Z_{0}\right)+Z_{1} \log _{10}\left(2 Z_{1}\right)+Z_{2} \log _{10}\left(4 Z_{2}\right)\right] .
\end{aligned}
$$

\section{Application to the WTCCC1 Type-2 Diabetes Data}

The HBD-GWAS strategy was applied to the WTCCC1 type-2 diabetes data [31] that consists of 1,924 individuals affected by type-2 diabetes and genotyped on Affymetrix 500K chips. They 


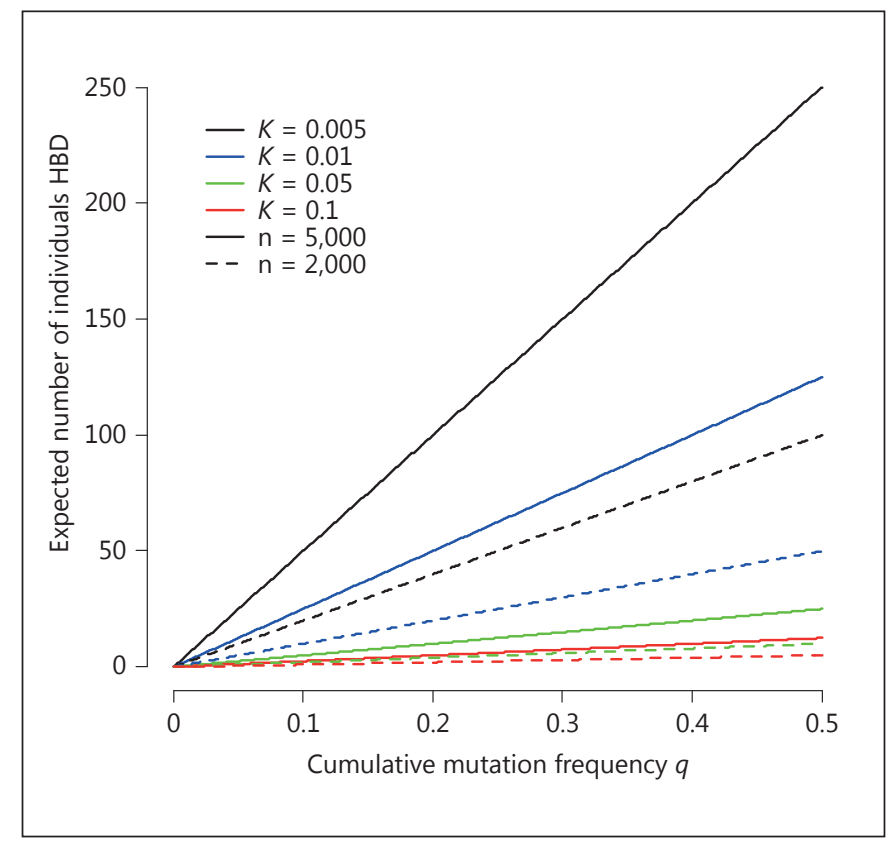

Fig. 1. Expected number of cases HBD for gene $G$ involved in disease $D$ in a sample of 5,000 cases (solid lines) or 2,000 cases (dashed lines). In the population, it is assumed that there is a proportion of inbred individuals of $\gamma=0.01$ with $\mathrm{F}=0.01$. The results are reported as a function of the cumulative frequency $q$ of disease-causing alleles in the gene and for different disease prevalence $K$.

were compared against two control datasets (58BC with 1,480 individuals and UKBS with 1,458 individuals). A stringent quality control was applied where only the SNPs with a call rate of at least 0.99 in any of the three datasets were kept. SNPs with a minor allele frequency below $1 \%$ and strongly $\left(\mathrm{p}<10^{-8}\right)$ departing from Hardy-Weinberg-expected proportions in the two control datasets were also excluded. A total of 283,942 SNPs were then available for the analyses. For FEstim, the marker allele frequencies were obtained from the control individuals. For FLOD, the disease allele frequency $\left(q^{\prime}\right)$ was set to 0.00001 . The ROHs were obtained with Plink [32] with default values, i.e. with a minimal ROH length of $1 \mathrm{Mb}$.

\section{Results}

\section{Properties of HBD-GWAS}

In figure 1 , the expected number of $\mathrm{HBD}$ cases in a sample of 2,000 cases and a sample of 5,000 cases is reported for a disease with varying disease prevalences $K$. Individuals were assumed to be from a population with $\gamma=1 \%$ inbred individuals with $\mathrm{F}=0.01$. Results are reported for values of $q$ between 0 and 0.5 . When $q$ is low, the expected number of HBD cases is very small and it increases with increasing values of $q$. This could seem counterintuitive, since among inbred individuals the proportion of individuals with two HBD alleles is expected to increase as the allele frequency decreases. However, this is compensated by the fact that, for a fixed disease prevalence, when $q$ decreases, the contribution of the rare variants to the disease within the gene becomes much reduced. For a disease with a prevalence $K=0.05$, similar to the prevalence of type- 2 diabetes in the UK, the number of HBD cases in a sample of 2,000 cases (that includes about 20 inbred individuals) reaches only 2 when $q=0.5$. This high value of $q$ is not possible (because of the limits on $q$ imposed by the prevalence), unless the number $(x)$ of rare variants in gene $G$ is high ( $x=$ at least 5$)$ and the disease model is TRUE_REC. This could correspond to a rather unrealistic situation where, for example, 10 fully penetrant rare recessive variants with a $5 \%$ frequency each (or 100 with a $0.5 \%$ frequency) are present in gene $G$ and increase the risk of the disease only when present at the homozygous state by a factor of $39\left(f_{0}=0.025, \mathrm{GRR}=\right.$ $39.07)$. For the same cumulative variant frequency $q$, the expected number of HBD cases, $H$, increases with decreasing prevalence: for $q=0.10$ in a sample of 5,000 cases, $H$ increases from 5 when $K=0.01$ to 10 when $K=$ 0.005 .

When the frequency $\gamma$ of inbred individuals in the sample population increases, the expected number of HBD cases also increases and consequently so does the evidence of linkage as measured by the EHFLOD. The value of $\mathrm{F}$ also has a strong impact on the number of HBD cases. All other parameters being equal, 5 times more HBD cases are expected with $\mathrm{F}=0.05$ than with $\mathrm{F}=0.01$ (table 1 ). Results are very similar under the two disease models. Indeed, $H$ is the same and only the penetrance, $f_{0}$, and the total number of inbred cases, $I$, vary between the two models but the variations are very limited unless $\gamma$ is very high. When $K$ is reduced from 0.05 to 0.01 , the EHFLOD is increased (e.g. for $\gamma=0.05$ and $\mathrm{F}=0.01, E H F L O D$ increases from 0.30 to 3.79 ). The HBD-GWAS strategy is thus expected to perform better for diseases with low prevalence since the contribution of the Mendelian subentities to the disease due to rare variants in gene $G$ is then more important. It also performs better when individuals have high inbreeding coefficients F. Indeed, while individuals with a low $\mathrm{F}$ are more informative for linkage as the lod-score is proportional to $1 / F$, their expected number in the sample of cases is proportional to $\mathrm{F}$ and thus decreases rapidly when $\mathrm{F}$ decreases. 
Table 1. Expected number $H$ of HBD cases and expected EHFLOD under the two disease models TRUE_REC and COMPO_REC in a sample of 2,000 cases for a disease with a prevalence of $K=1 \%$ or $K=5 \%$ and various values of the proportion $\gamma$ of inbred individuals and of the inbreeding coefficient $\mathrm{F}$

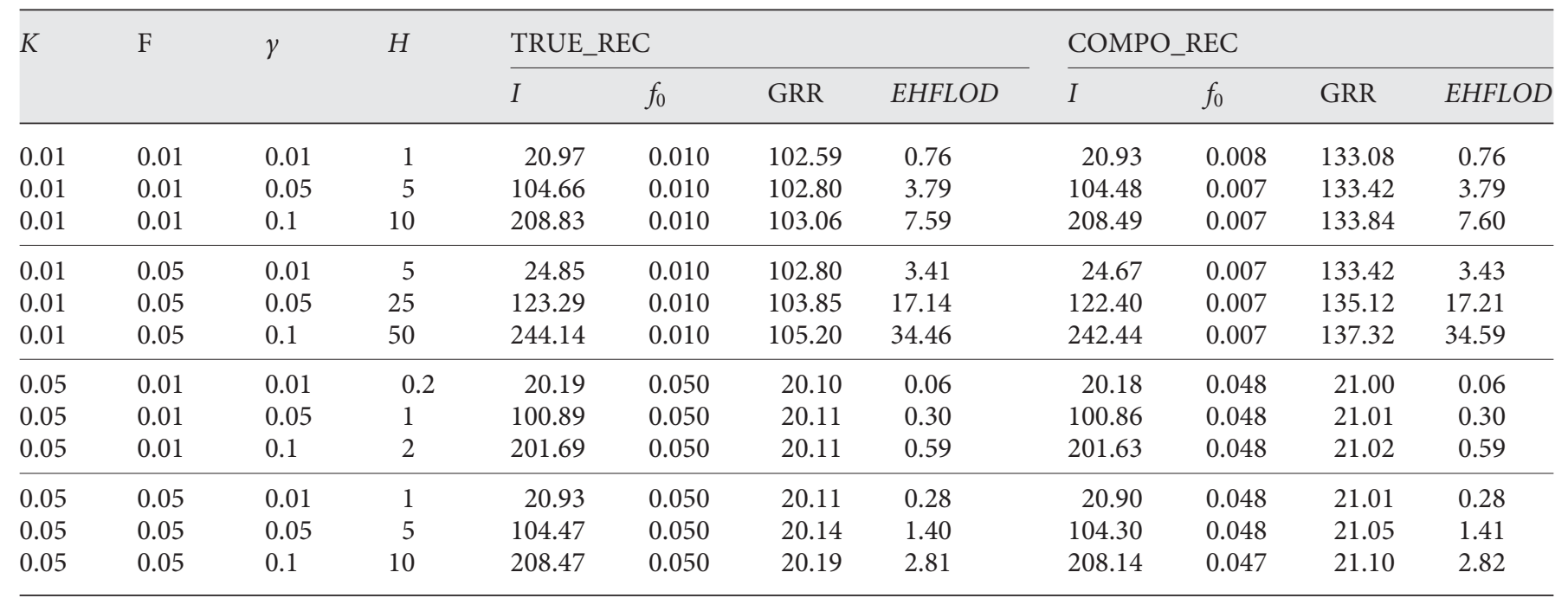

It is assumed that the number of fully penetrant recessive variants in the gene is $x=5$ and their cumulative frequency is $q=0.05 . I=$ Expected number of inbred individuals among the cases; $f_{0}=$ penetrance of non-homozygous variant carriers; GRR = corresponding genotype relative risk.

\section{Expected Power of HBD-GWAS Compared to ASP}

To compare the linkage evidence provided by inbred affected individuals and by ASP, we computed their expected power for different values of the parameters under the two disease models (fig. 2), considering a population with $\gamma=1 \%$ of inbred individuals and $\mathrm{F}=0.05$.

The only situations where the power of the HBDGWAS strategy exceeds the power of the linkage strategy on ASP are situations where $q$ is low and the number $(x)$ of variants is high. Indeed, under the TRUE_REC model, the ASP design performs poorly when the level of allelic heterogeneity measured by the number $(x)$ of variants increases, whereas the HBD-GWAS strategy is not affected by allelic heterogeneity. However, in these situations where EHFLOD > EMLS, the power to detect linkage is extremely low (below 10\%). Under the COMPO_REC model, the performances of the ASP design are no longer affected by allelic heterogeneity because some evidence of linkage is provided by the composite heterozygous genotypes, i.e. those from genotype category 2 with two different mutant alleles.

If we assume that the proportion of inbred individuals in the sample is increased $(\gamma=0.05$ and $\mathrm{F}=0.05$, for example; table 2), we can find some situations where inbred cases could provide much more information on linkage than ASP provided the sample of cases is large $(n=5,000)$.

\section{Application to the WTCCC1 Type-2 Diabetes Data}

The results of the HBD-GWAS method applied to the WTCCC1 type-2 diabetes data are shown in figure 3. Among the affected individuals, $17(0.9 \%)$ had a median estimated inbreeding coefficient $>0.01$ (see online suppl. table S1 for the individual values; www. karger.com/doi/10.1159/000346790) and were further considered for the computation of HFLOD. A similar proportion of inbred individuals was also observed among controls $(20(0.7 \%)$ of the 2,938 controls has a median $\mathrm{F} \geq 0.01,13$ among the 1,458 UKBS controls and 7 among the 1,480 58BC controls). Values of HFLOD $>2$ were observed on chromosomes 1,13 and 14 (fig. 4a) with a maximum of 2.6 reached on chromosome 1 . In this region, there are 3 individuals (WTCCC62035, WTCCC166968, WTCCC167708) showing long overlapping ROHs (length 18.7, 26, and $30.2 \mathrm{Mb}$, respectively), within which 2 additional individuals (WTCCC62040, WTCCC60660) share short ROHs, reducing the region shared by the 5 individuals to $1.6 \mathrm{Mb}$ (fig. $4 \mathrm{~b}$ ). Interestingly, this smaller shared region contains only one gene, NEGR1, for which a $45-\mathrm{kb}$ deletion and rare mutant alleles have been associated with obesity [33]. None of the 20 inbred controls has an $\mathrm{ROH}$ in this region and HFLOD results on chromosome 1 were robust to disease allele frequency changes ( when $q^{\prime}=0.01$, maximum HFLOD $=2.5$ ). 


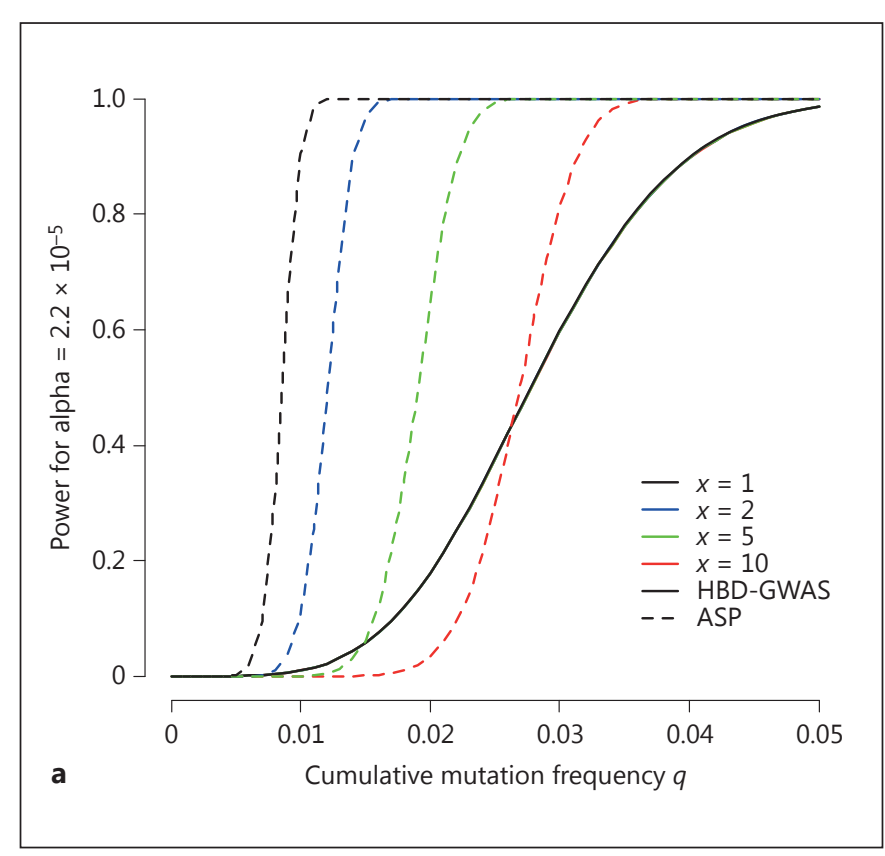

Fig. 2. Expected power of the HBD-GWAS strategy applied on inbred cases from a sample of 5,000 cases (solid lines) and of the non-parametric lod-score on 300 ASP (dashed lines). The disease prevalence is $K=0.01$, and there is a proportion of inbred individuals of $\gamma=0.01$ with $\mathrm{F}=0.05$ in the population. The results are

\section{Discussion}

The selection of inbred cases has proven to be a good strategy to evidence rare recessive variants involved in monogenic diseases by homozygosity mapping. In order to determine if this could also be used in the context of complex diseases to identify some monogenic-like subentities of these diseases that could be due to rare recessive variants, we performed some theoretical computations to evaluate the power of this strategy. We found that, under most of the scenarios investigated, it will not be possible to evidence these hidden subentities by selecting inbred individuals from samples of cases genotyped for GWAS. Indeed, the monogenic subentities would explain too little of the disease prevalence to be represented enough in the sample of cases. It could work for complex diseases with low prevalence provided large samples are available. In this situation of low prevalence, however, such monogenic subentities should be easily detectable by looking at allele sharing among ASP. Indeed, even if ASP linkage studies have often been limited by rather small sample sizes compared to GWAs studies, we confirm that even with a few hundred ASP, the power achieved exceeds the power of the HBD-

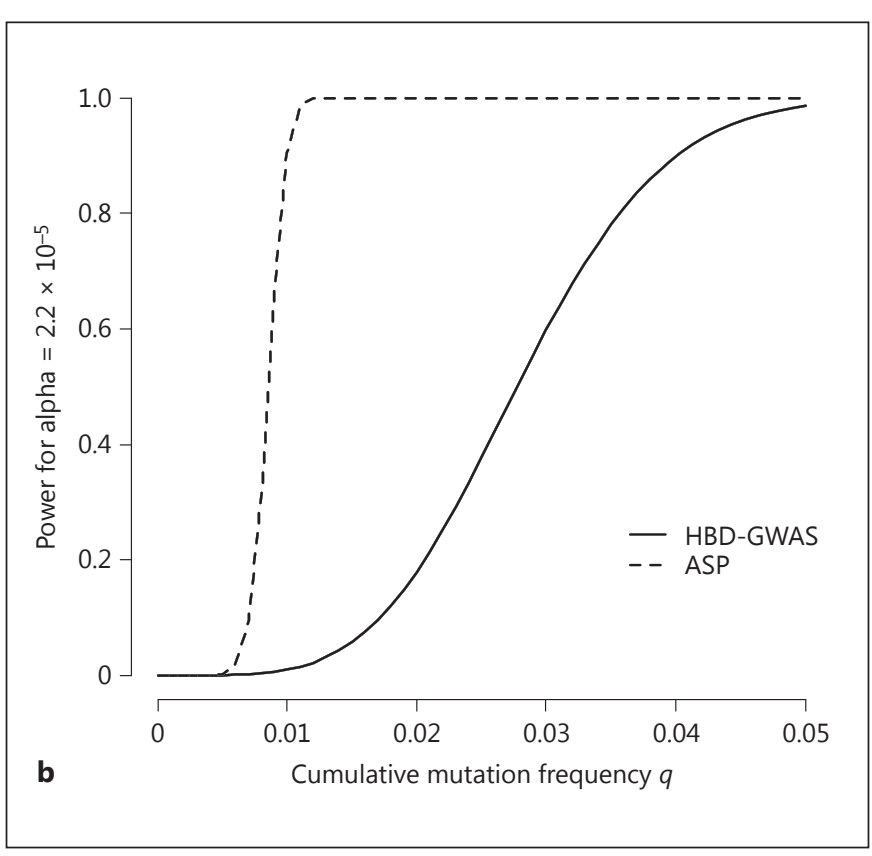

reported as a function of the cumulative frequency $q$ of diseasecausing alleles in the gene and for different numbers $(x)$ of mutant alleles under the true recessive model (a) and the composite recessive model (b).

GWAS strategy. This is of course especially true under the COMPO_REC model as the HBD-GWAS strategy is only able to detect the true recessive. Inbred cases perform better than ASP only in the situation of strong heterogeneity where several rare variants within the gene can be involved.

The poor performances of inbred cases to detect rare recessive variants involved in complex traits contrast with the different studies in the literature that advocate the search for regions of HBD in patients to evidence rare recessive variants. It should be noted, however, that most of these studies did not evaluate the power of these strategies and, when applied to real data, some were successful (see for example $[22,34,35]$ ) but several also failed (see for example [36-38]). In the study by Lencz et al. [22], where highly penetrant recessive loci were evidenced in schizophrenia for the first time by a whole-genome homozygosity association strategy, the authors only focused on common $\mathrm{ROH}$. Indeed, they only considered those ROHs shared by at least 10 subjects, and most of them were found both in cases and controls. It is thus unlikely that they contain very rare recessive variants that are fully penetrant and could represent the kind of Mendelian subentities we were seeking here. 

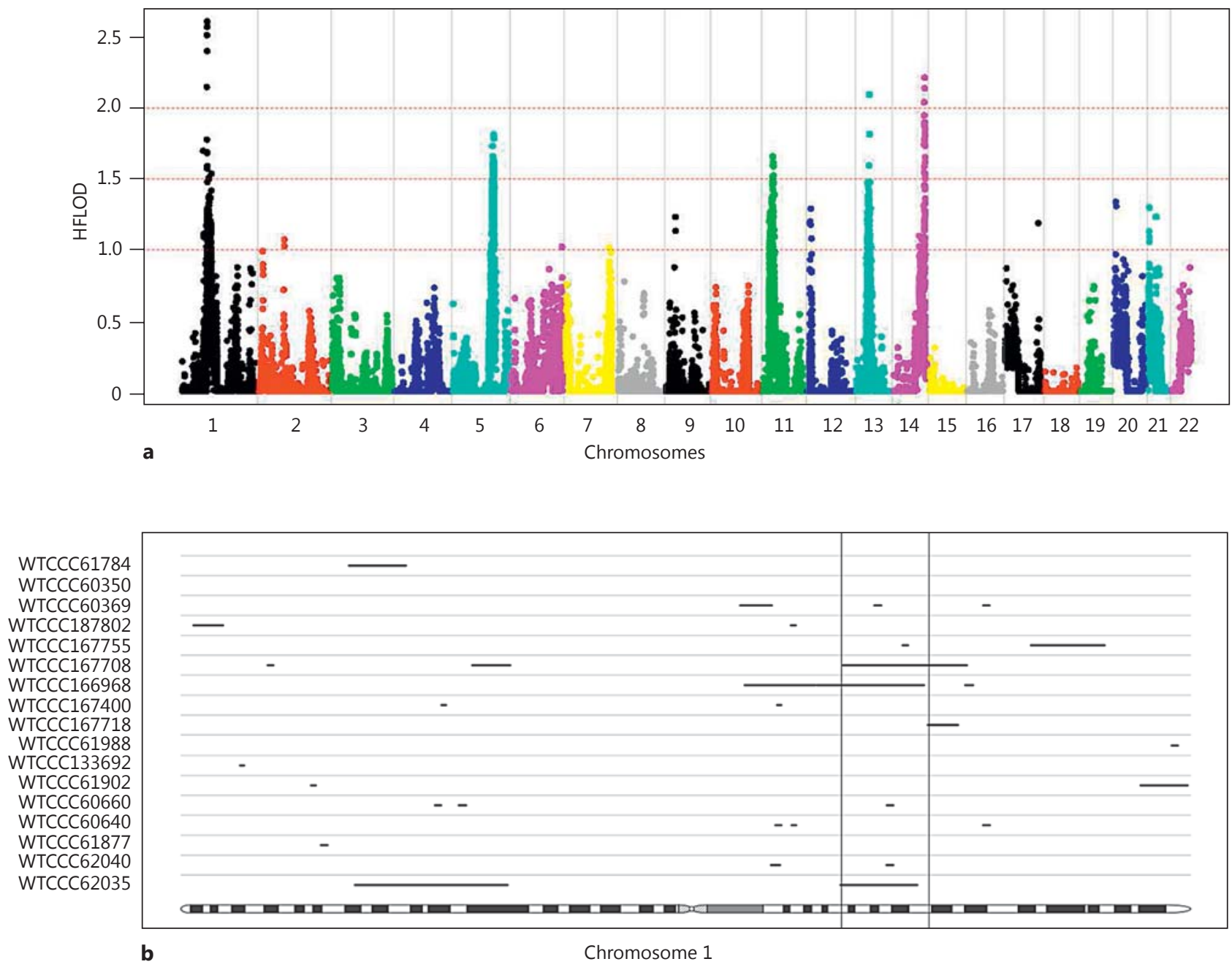

Fig. 3. The HBD-GWAS method applied to the WTCCC1 type-2 diabetes data. a HFLOD over the genome and b the ROHs on chromosome 1 for the 17 inbred individuals with $\mathrm{F} \geq 0.01$. The vertical lines on the ROHs delimit the region where HFLOD $\geq 1$.

More recently, a study by Chahrour et al. [39] was successful in identifying some interesting genes with homozygous mutations potentially involved in autism, in ROHs detected using SNP data on 1,000 families (5,431 individuals) from the Autism Genetic Research Exchange (AGRE). Exome sequencing of 16 of the patients who showed longer ROHs than the others revealed potentially pathogenic recessive mutations that segregated perfectly with the disease in 4 out of the 16 families. The availability of family data was, however, a key point to help select the interesting mutations. Moreover, the affected indi- viduals from AGRE are not random autistic cases, but probands in families with at least two affected individuals. Selecting multiplex families is a way to enrich the sample in Mendelian subentities of the disease and could explain the success of homozygosity mapping in this example.

The Early Onset Parkinson Disease (EOPD) study has also recently evidenced an excess of homozygosity in patients compared to controls. Using two different homozygosity mapping approaches, Simón-Sánchez et al. [40] found one region on the short arm of chromosome 19, where 6 different cases from 4 different countries con- 
Table 2. Comparison of the evidence of linkage provided by inbred cases and ASP for different numbers $(x)$ of fully penetrant recessive variants

\begin{tabular}{|c|c|c|c|c|c|c|c|c|c|c|}
\hline Disease model & $\mathrm{n}$ & $x$ & $f_{0}$ & GRR & $I$ & $H$ & EHFLOD & Power of HBD & EMLS & Power of ASP \\
\hline \multirow[t]{5}{*}{ TRUE_REC } & \multirow[t]{3}{*}{5,000} & 1 & 0.048 & 21.05 & 260.75 & 12.50 & 3.52 & 0.4753 & 5.54 & 0.7760 \\
\hline & & 2 & 0.049 & 20.54 & 261.01 & 12.50 & 3.51 & 0.4747 & 1.84 & 0.0667 \\
\hline & & 10 & 0.050 & 20.14 & 261.23 & 12.50 & 3.51 & 0.4742 & 0.09 & 0.0001 \\
\hline & \multirow[t]{2}{*}{2,000} & 1 & 0.048 & 21.05 & 104.30 & 5.00 & 1.41 & 0.0617 & 5.54 & 0.7760 \\
\hline & & 10 & 0.050 & 20.14 & 104.49 & 5.00 & 1.40 & 0.0615 & 0.09 & 0.0001 \\
\hline \multirow{5}{*}{ COMPO_REC } & \multirow[t]{4}{*}{5,000} & 1 & 0.048 & 21.05 & 260.75 & 12.50 & 3.52 & 0.4753 & 5.54 & 0.7760 \\
\hline & & 2 & 0.048 & 21.05 & 260.75 & 12.50 & 3.52 & 0.4753 & 5.54 & 0.7769 \\
\hline & & 5 & 0.048 & 21.05 & 260.75 & 12.50 & 3.52 & 0.4753 & 5.54 & 0.7770 \\
\hline & & 10 & 0.048 & 21.05 & 260.75 & 12.50 & 3.52 & 0.4753 & 5.54 & 0.7770 \\
\hline & 2,000 & 10 & 0.048 & 21.05 & 104.30 & 5.00 & 1.41 & 0.0617 & 5.54 & 0.7770 \\
\hline
\end{tabular}

The cumulative variant frequency $q$ is 0.05 . Samples of 2,000 or 5,000 cases and 300 ASP are considered. The disease prevalence is $5 \%$ and there is a proportion of inbred individuals $\gamma=0.05$ with $\mathrm{F}=0.05$ in the population. The power of the two designs is also reported for a nominal type-one error rate of $2.2 \times 10^{-5}$. GRR = Genotype-relative risk; $I=$ expected number of inbred individuals among the cases; $H=$ expected number of HBD cases.

tained rare overlapping ROHs. Exome sequencing of these samples failed, however, to find any variant associated with the disease. The authors proposed several possible explanations for their results including the possibility that, rather than highly penetrant mutations, there might in fact exist a burden of multiple low-penetrance homozygous alleles in cases. This is an alternative model to the one investigated here. It is possible that the HBDGWAS strategy could perform better under such a model and it would thus be interesting to study it further.

Coming back to the type- 2 diabetes application, it is interesting to note that within the chromosome 1 region where the HFLOD was maximal, it is possible to delimit a small subregion where as many as 5 cases have overlapping ROHs. This number is higher than the number of HBD cases expected in our model for a disease with a prevalence of $5 \%$. However, the theoretical computations were done assuming that all inbred cases have the same inbreeding coefficient and this is not true in the WTCCC1 type-2 diabetes sample. Here some of the cases have inbreeding coefficients of 5\% and above. It is thus possible that this region and the 5 patients identified carry some rare recessive variants that could fully explain the disease in these patients. It is also possible that, in fact, these patients carry the common $45-\mathrm{kb}$ deletion upstream of the NEGR1 gene that was found associated with obesity [33] and that the region appears homozygous only because one of the two copies of this genomic region is missing. Even if this cannot be completely excluded, it is, however, unlikely that the $45-\mathrm{kb}$ deletion could mimic $\mathrm{ROHs}$ of several Mbs. Even if our theoretical derivations bring pessimism regarding the possible success of the proposed strategy and even if the maximum lod-score achieved is rather low, it might still be worth investigating further the chromosome 1 region highlighted here.

The theoretical derivations presented in this paper were performed to study the impact of the different parameters on the performance of homozygosity mapping. They allow determining what power could be achieved in best-case scenarios. In these derivations, we only consider situations where all inbred individuals have the same inbreeding coefficient and all variants within the gene have the same frequency. This is not realistic as in real data applications, inbred individual will each have a different inbreeding coefficient and the different variants within a gene will have different frequencies. The equa- 
tions we provide can, however, also be used to study more complex scenarios and estimate the expected power for a given sample with its own characteristics.

Another limitation of our derivation is the fact that we assume that the inbreeding coefficients are known. In practice, however, one will have to estimate inbreeding coefficients first using the genetic data and work with these estimates rather than with true values. This could lead to some reduction in power. It is also possible that some patients who are not inbred get inbreeding coefficient estimates different from zero. Including these patients in the analysis could lead to some power losses and this is the reason, why we chose to only include patients with inbreeding coefficient estimates greater than $1 \%$. Through simulations [S. Gazal, unpubl. results], we found that most of these patients are expected to be truly inbred. The choice of this threshold, however, might be important as it determines the number of cases who will be included in the homozygosity mapping step. Depending on the sample, the characteristics of the studied population and the marker informativity, other thresholds may be optimal.

Another problem in practical applications is the presence of homozygous deletions that could be confound- ed with segments of homozygosity. Investigators would need to be aware of this problem and double-check whether any region of homozygosity found shared by patients contains large deletions. For that purpose, an easy solution consists in searching databases for reported deletions in the regions identified. Novel deletions will, however, be missed with this strategy; an alternative strategy is to use copy-number calling algorithms on the SNP chip intensity data to determine if the studied patients have some deletions in the regions of interest. Several algorithms have been developed recently to detect copy-number variations and might be used provided raw SNP chip data are available (for a comparison of algorithms, see Marenne et al. [41]).

\section{Acknowledgements}

This study makes use of data generated by the Wellcome Trust Case Control Consortium. A full list of the investigators who contributed to the generation of the data is available from www.wtccc. org.uk. We thank two anonymous reviewers for their fruitful comments.

\section{References}

1 Manolio TA, Collins FS, Cox NJ, et al: Finding the missing heritability of complex diseases. Nature 2009;461:747-753.

-2 Bodmer W, Bonilla C: Common and rare variants in multifactorial susceptibility to common diseases. Nat Genet 2008;40:695701.

3 Zhu X, Feng T, Li Y, Lu Q, Elston RC: Detecting rare variants for complex traits using family and unrelated data. Genet Epidemiol 2010; 34:171-187.

4 Feng T, Zhu X: Genome-wide searching of rare genetic variants in WTCCC data. Hum Genet 2010;128:269-280.

5 Browning SR, Thompson EA: Detecting rare variant associations by identity-by-descent mapping in case-control studies. Genetics 2012;190:1521-1531.

-6 Houwen RH, Baharloo S, Blankenship K, Raeymaekers P, Juyn J, Sandkuijl LA, Freimer NB: Genome screening by searching for shared segments: mapping a gene for benign recurrent intrahepatic cholestasis. Nat Genet 1994;8:380-386.

-7 Bourgain C, Genin E, Holopainen P, Mustalahti K, Maki M, Partanen J, Clerget-Darpoux F: Use of closely related affected individuals for the genetic study of complex diseases in founder populations. Am J Hum Genet 2001; 68:154-159.
-8 Browning BL, Browning SR: A fast, powerful method for detecting identity by descent. Am J Hum Genet 2011;88:173-182.

$\checkmark 9$ Wilkie AO: The molecular basis of genetic dominance. J Med Genet 1994;31:89-98.

10 Jimenez-Sanchez G, Childs B, Valle D: Human disease genes. Nature 2001;409:853-855.

11 Furney SJ, Alba MM, Lopez-Bigas N: Differences in the evolutionary history of disease genes affected by dominant or recessive mutations. BMC Genomics 2006; 7:165.

12 Blekhman R, Man O, Herrmann L, Boyko AR, Indap A, Kosiol C, Bustamante CD, Teshima KM, Przeworski M: Natural selection on genes that underlie human disease susceptibility. Curr Biol 2008;18:883-889.

13 Lettre G, Lange C, Hirschhorn JN: Genetic model testing and statistical power in population-based association studies of quantitative traits. Genet Epidemiol 2007;31:358-362.

14 Lander ES, Botstein D: Homozygosity mapping: a way to map human recessive traits with the DNA of inbred children. Science 1987;236:1567-1570

15 Génin E, Todorov AA: Homozygosity Mapping: Encyclopedia of Life Science. Chichester, John Wiley and Sons Ltd., 2007.
16 Leutenegger AL, Labalme A, Genin E, Toutain A, Steichen E, Clerget-Darpoux F, Edery P: Using genomic inbreeding coefficient estimates for homozygosity mapping of rare recessive traits: application to TaybiLinder syndrome. Am J Hum Genet 2006;79: 62-66.

17 Leutenegger AL, Prum B, Genin E, Verny C, Lemainque A, Clerget-Darpoux F, Thompson EA: Estimation of the inbreeding coefficient through use of genomic data. Am J Hum Genet 2003;73:516-523.

18 Morton NE: Sequential tests for the detection of linkage. Am J Hum Genet 1955;7: 277-318.

19 Edery P, Marcaillou C, Sahbatou M, et al: Association of TALS developmental disorder with defect in minor splicing component U4atac snRNA. Science 2011;332:240243.

20 Gibbs JR, Singleton A: Application of genome-wide single nucleotide polymorphism typing: simple association and beyond. PLoS Genetics 2006;2:e150.

21 Howrigan DP, Simonson MA, Keller MC: Detecting autozygosity through runs of homozygosity: a comparison of three autozygosity detection algorithms. BMC Genomics 2011;12: 460.
Rare Recessive Variant Detection in GWAS Data
Hum Hered 2012;74:142-152 DOI: $10.1159 / 000346790$ 
-22 Lencz T, Lambert C, DeRosse P, Burdick KE, Morgan TV, Kane JM, Kucherlapati R, Malhotra AK: Runs of homozygosity reveal highly penetrant recessive loci in schizophrenia. Proc Natl Acad Sci USA 2007;104:19942-19947.

23 Wang S, Haynes C, Barany F, Ott J: Genomewide autozygosity mapping in human populations. Genet Epidemiol 2009;33:172-180.

24 Zhang L, Yang W, Ying D, Cherny SS, Hildebrandt F, Sham PC, Lau YL: Homozygosity mapping on a single patient: identification of homozygous regions of recent common ancestry by using population data. Hum Mutat 2011;32:345-353.

25 Leutenegger AL, Sahbatou M, Gazal S, Cann $\mathrm{H}$, Genin E: Consanguinity around the world: ehat do the genomic data of the HGDP-CEPH diversity panel tell us? Eur J Hum Genet 2011; 19:583-587.

26 Xing C, Morris N, Xing G: Distribution of model-based multipoint heterogeneity lod scores. Genet Epidemiol 2010;34:912-916.

27 Génin E, Clerget-Darpoux F: Consanguinity and the sib-pair method: an approach using identity by descent between and within individuals. Am J Hum Genet 1996;59:1149-1162.

28 Lander E, Kruglyak L: Genetic dissection of complex traits: guidelines for interpreting and reporting linkage results. Nat Genet 1995; 11:241-247.
9 Risch N: Linkage strategies for genetically complex traits. III. The effect of marker polymorphism on analysis of affected relative pairs. Am J Hum Genet 1990;46:242-253.

30 Poznik GD, Adamska K, Xu X, Krolewski AS, Rogus JJ: A novel framework for sib pair linkage analysis. Am J Hum Genet 2006;78:222230.

31 WTCCC: Genome-wide association study of 14,000 cases of seven common diseases and 3,000 shared controls. Nature 2007;447:661678.

32 Purcell S, Neale B, Todd-Brown K, et al: Plink: a tool set for whole-genome association and population-based linkage analyses. Am Hum Genet 2007;81:559-575.

33 Willer CJ, Speliotes EK, Loos RJ, et al: Six new loci associated with body mass index highlight a neuronal influence on body weight regulation. Nat Genet 2009;41:25-34.

34 Yang TL, Guo Y, Zhang LS, Tian Q, Yan H, Papasian CJ, Recker RR, Deng HW: Runs of homozygosity identify a recessive locus 12q21.31 for human adult height. J Clin Endocrinol Metab 2010;95:3777-3782.

35 Casey JP, Magalhaes T, Conroy JM, et al: A novel approach of homozygous haplotype sharing identifies candidate genes in autism spectrum disorder. Hum Genet 2011;131: 565-579.
36 Hosking FJ, Papaemmanuil E, Sheridan E, et al: Genome-wide homozygosity signatures and childhood acute lymphoblastic leukemia risk. Blood 2010;115:4472-4477.

37 Kuningas M, McQuillan R, Wilson JF, Hofman A, van Duijn CM, Uitterlinden AG, Tiemeier H: Runs of homozygosity do not influence survival to old age. PloS One 2011; 6:e22580.

38 Sims R, Dwyer S, Harold D, et al: No evidence that extended tracts of homozygosity are associated with Alzheimer's disease. Am J Med Genet B Neuropsychiatr Genet 2011; 156B:764-771.

39 Chahrour MH, Yu TW, Lim ET, et al: Wholeexome sequencing and homozygosity analysis implicate depolarization-regulated neuronal genes in autism. PLoS Genetics 2012; 8:e1002635.

40 Simón-Sánchez J, Kilarski LL, Nalls MA, et al: Cooperative genome-wide analysis shows increased homozygosity in early onset Parkinson's disease. PloS One 2012;7:e28787.

41 Marenne G, Rodriguez-Santiago B, Closas MG, et al: Assessment of copy number variation using the Illumina Infinium 1M SNP-array: a comparison of methodological approaches in the Spanish Bladder Cancer/EPICURO study. Hum Mutat 2011;32:240-248. 